\title{
Konus-Wind observations of the new soft gamma-ray repeater SGR $0501+4516$
}

\author{
R. L. Aptekar ${ }^{1}$, T. L. Cline ${ }^{2}$, D. D. Frederiks ${ }^{1}$, S. V. Golenetskii ${ }^{1}$, E. P. Mazets ${ }^{1}$, V. D. Pal'shin ${ }^{1}$
}

\begin{abstract}
In 2008 August, the new soft gamma-ray repeater SGR 0501+4516 was discovered by Swift. The source was soon confirmed by several groups in space- and ground-based multi-wavelength observations. In this letter we report the analysis of five short bursts from the recently discovered SGR, detected with Konus-Wind gamma-ray burst spectrometer. Properties of the time histories of the observed events, as well as results of multi-channel spectral analysis, both in the 20-300 keV energy range, show, that the source exhibits itself as a typical SGR. The bursts durations are $\lesssim 0.75 \mathrm{~s}$ and their spectra above $20 \mathrm{keV}$ can be fitted by optically-thin thermal bremsstrahlung (OTTB) model with $k T_{O T T B}$ of $20-40 \mathrm{keV}$. The spectral evolution is observed, which resembles the SGR 1627-41 bursts, where a strong hardness-intensity correlation was noticed in the earlier Konus-Wind observations. The peak energy fluxes of all five events are comparable to highest those for known SGRs, so a less distant source is implied, consistent with the determined Galactic anti-center direction. Supposing the young supernova remnant HB9 (at the distance of $1.5 \mathrm{kpc}$ ) as a natal environment of the source, the peak luminosities of the bursts are estimated to be $(2-5) \times 10^{40} \mathrm{erg} \mathrm{s}^{-1}$. The values of the total energy release, given the same assumptions, amount to $(0.6-6) \times 10^{39} \mathrm{erg}$. These estimations of both parameters are typical for short SGR bursts.
\end{abstract}

Subject headings: gamma rays: bursts - gamma rays: observations - pulsars: individual (SGR 0501+4516) - stars: neutron

\section{INTRODUCTION}

Soft gamma-ray repeaters (SGRs) were discovered almost 30 years ago (Mazets et al. 1979a, b). Two main types of bursting emission are observed. The active stage of the SGRs consists of many short $\left(<1\right.$ second) bursts of hard X-rays with energy release in the $10^{39}-10^{41}$ erg range. This activity may last from several days up to over a year, followed by a quiescent period of up to several years. The less frequent SGR activity consists of the rare, giant flare, with an initial pulse having energy release as high as $2 \times 10^{46} \mathrm{erg}$ (Frederiks et al. 2007a) followed by a decaying pulsating tail. At most, only one such giant flare has yet been observed per SGR source in the $>30$ years of

\footnotetext{
${ }^{1}$ Ioffe Physico-Technical Institute of the Russian Academy of Sciences, St. Petersburg, 194021, Russia

${ }^{2}$ Emeritus: NASA's Goddard Space Flight Center, Greenbelt, MD 20771, USA
} 
total monitoring. All SGRs are now known to be rapidly slowing X-ray pulsars with periods of 2 to 8 seconds and luminosities of $L \sim 10^{35} \mathrm{erg} \mathrm{s}^{-1}$. Sharing these characteristics with anomalous X-ray pulsars (AXPs), the sources are believed to form a rare class of strongly magnetized isolated neutron stars (NS); see Mereghetti (2008) for a recent review. Under the assumption of pure magnetic dipole braking, the values of their period derivative $\left(\sim 10^{-13}-10^{-10} \mathrm{~s} \mathrm{~s}^{-1}\right)$ imply an enormous magnetic field strength of $\sim 10^{14}-10^{15} \mathrm{G}$, which is orders of magnitude larger than those of the radio pulsars. These extreme magnetic fields are supposed to be an ultimate source of energy for the bright persistent X-ray emission and the bursting activity of the SGRs and the AXPs.

Only four SGR sources were definitely known, with only one other possibility, prior to $\mathrm{Au}-$ gust 22, 2008, when the Swift team reported the discovery of the new soft gamma-ray repeater SGR 0501+4516 (Holland et al. 2008; Barthelmy et al. 2008). Swift observed two repeated bursts, localizing their fading X-ray counterpart to an area of 2.6 arcsec radius in the direction of the Galactic anti-center. The SGR nature of this source was confirmed by the discovery of the $\sim 5.76$ s X-ray pulsar (Gogus et al. 2008) in the Swift-XRT localization circle. The pulsar spindown rate $\dot{P}=1.5(5) \times 10^{-11} \mathrm{~s} \mathrm{~s}^{-1}$ and the inferred dipole magnetic field of $B=3 \times 10^{14} \mathrm{G}$ were soon estimated (Woods et al. 2008). The refined phase-coherent solution for the combined $X M M$-Newton, Suzaku-XIS, and Swift-XRT data sets yields the best fit $P=5.7620695(1) \mathrm{s}, \dot{P}=$ $6.7(1) \times 10^{-12} \mathrm{~s} \mathrm{~s}^{-1}$, and $\ddot{P}=-1.6(4) \times 10^{-19} \mathrm{~s} \mathrm{~s}^{-2}$ (for the reference epoch MJD 54701.0) (Rea et al. 2009). Multi-wavelength observations reported possible near-infrared (Tanvir \& Varricatt 2008; Rea et al. 2008a) and optical (Fatkhullin et al. 2008) counterparts for the SGR. The continuing SGR activity was observed by several GRB instruments (Palmer et al. 2008a; Golenetskii et al. 2008; Kouveliotou et al. 2008) to have declined by the end of August, 2008 (Palmer et al. 2008b).

During this period the Konus GRB spectrometer onboard the Wind spacecraft Aptekar et al. 1995) detected five SGR 0501+4516 bursts that were sufficiently intense to enable the triggered observation mode. Observations of the light curves, spectral analyses and the estimated energies of these events are presented here, together with comparisons against known SGR behavior previously observed with the same Konus-Wind instrument.

\section{OBSERVATIONS}

The initial SGR 0501+4516 bursts reported by Swift on August 23, 2008 were too weak to enable the Konus-Wind triggered mode, which is optimized for GRB detection. However, the first triggered Konus event from that source took place on the same day at 04:47:46.194 s UT. The next four Konus bursts (see Table 1) were detected within few days, with the last triggered event on August 26th.

The light curves for these five bursts are shown in Figure 1 with 2 ms resolution. The energy ranges differ in the figures to conform to the available observations, with the detector's energy 
thresholds at $20 \mathrm{keV}, 70 \mathrm{keV}$, and $300 \mathrm{keV}$. Thus, the profile for the $080823 \mathrm{a}$ event is for 70 to $300 \mathrm{keV}$, whereas the other four profiles are for 20 to $300 \mathrm{keV}$. No emission was detected above $300 \mathrm{keV}$. Dead time corrections of up to $50 \%$ have been made. It is clear that the bursts are short $(\lesssim 0.75 \mathrm{~s})$ events and have a diverse temporal behavior with multiple peaks. The start times, total durations, and peak times of the bursts are given in Table 1 ,

For each of these events, up to four energy spectra were measured with $64 \mathrm{~ms}$ accumulation times, as indicated in the event profiles in Figure1, illustrated with dashed lines. The raw count-rate spectra were rebinned in order to have at least 20 counts per energy bin, and fitted using XSPEC, version 11.3 (Arnaud 1996), using only the 20 to $200 \mathrm{keV}$ fitting interval since no emission was detected at a higher energy. Results of the spectral analyses are summarized in Table 2.

A good fit was obtained for the initial, intense phase to a power-law with an exponential cutoff (CPL) model, $d N_{p h} / d E \propto E^{-\alpha} \exp \left[-(2-\alpha) E / E_{p}\right]$, where $E_{p}$ is the peak energy in the $E F(E)$ spectrum. The spectral index values $\alpha$ do not differ far from 1 , so the optically-thin thermal bremsstrahlung (OTTB) model $d N_{p h} / d E \propto E^{-1} \exp [-E / k T]$ can be used as well, which is often considered for SGR spectra above $15 \mathrm{keV}$. In both cases the values of $E_{p}$ (or $k T$ ) lie in the 20 to $45 \mathrm{keV}$ range. Single and dual black-body spectral models were also tested, but they are too steep at higher energies to provide a good agreements to the data above $100 \mathrm{keV}$.

The spectral evolution of the events can be demonstrated, making use of the hardness ratio of counts in the two available energy windows, G1 (20-70 keV) and G2 (70-300 keV). The behavior of the G2/G1 hardness ratio and the $k T_{O T T B}$ spectral parameter, the value of which is estimated using a detector response matrix, is plotted in Figure 2 for the 080824 burst, together with its two channel light curves. A hardness-intensity correlation is apparent for all observed bursts, and the range of the $k T_{O T T B}$ variation is in a good agreement with the one derived from the multichannel spectra.

Using the observed event profiles and spectral analyses, the total energy fluences and the $2 \mathrm{~ms}$ peak fluxes in the 20 to $200 \mathrm{keV}$ range were determined, as summarized in Table 1. All errors given in Tables 1 and 2 are at the $90 \%$ confidence level.

\section{DISCUSSION}

The Konus-Wind omnidirectional detector array constantly observes the whole sky in a wide energy range from $\sim 20 \mathrm{keV}$ to $\sim 14 \mathrm{MeV}$. In interplanetary space far outside the Earth's magnetosphere, Konus has the advantages over Earth-orbiting GRB monitors of continuous coverage, uninterrupted by Earth occultation, and a steady background, undistorted by passages through the Earth's trapped radiation. Therefore, it has had the opportunity to observe almost all intense bursts from the known Galactic soft gamma-ray repeaters (SGR 1806-20, SGR 1900+14, and SGR 1627-41) while in their active states (Aptekar et al. 2001), during the last 14 years of its continuous operation. Thus, use of the same instrument provides the optimum basis for comparison 
of the newly discovered SGR 0501+4516 activity.

The durations, the profiles, and the energy spectra of the SGR 0501+4516 bursts are all typical for short SGR bursts. The spectral evolution observed in these events is not in common with SGR 1806-20 or SGR 1900+14 (Aptekar et al. 2001), but resembles the SGR 1627-41 bursts, where a strong hardness-intensity correlation was noticed in the Konus-Wind observations (Mazets et al. 1999). In contrast, a hardness-intensity anti-correlation was found in some weak short bursts as well as throughout the overall burst sample from SGR 1806-20 observed with INTEGRAL (Götz et al. 2004, 2006). Among 55 bursts from SGR 1806-20 and SGR 1900+14 observed by HETE-2 only for 3 bursts was a clear (but moderate) hardness-intensity anti-correlation reported; for the other 3 bursts, a hint of a hard component at end of the burst was found (Nakagawa et al. 2007). However, it should be noted that most of the HETE-2 and INTEGRAL bursts are much weaker than the Konus-Wind trigger bursts. A similar anti-correlation was found in two peculiar hard intense bursts from SGR 1900+14 (Woods et al. 1999). The mechanism responsible for the observed spectral evolution is not yet established.

Since the short bursts from SGR 1627-41 and SGR 0501+4516 are similar to those from other SGRs in terms of their characteristics such as light curve shapes, durations, and spectra, they are thus very likely caused by the same physical process 1 , and the strong spectral evolution observed in these two SGRs may be due to some specific characteristics of the sources (e.g. magnetic field strength and configuration).

The one outstanding feature in the SGR $0501+4516$ bursts phenomenology is the relatively high intensity. The values of the energy fluence for these events are typically greater than average among the known Galactic SGRs. Even more distinctive features are the average peak fluxes $\left(\sim 10^{-4} \mathrm{erg} \mathrm{cm}^{-2} \mathrm{~s}^{-1}\right)$ of the five detected events, at the maximum range exhibited by all the other SGR bursts observed (Figure 3).

The SGR bursts fluences tend to follow a power law distribution (Göğüs et al. 2000; Aptekar et al. 2001; Götz et al. 2006). However, the exact shape and parameters of the observed fluences and peak fluxes distributions are not well determined, and may be subject to a detection-specific bias. Therefore, model-independent nonparametric methods were used to test the assumption that SGR $0501+4516$ bursts peak fluxes are distributed quantitatively in the same way as those from the other SGRs detected by Konus-Wind. This hypothesis is rejected for all three SGR's bursts sets at the 0.05 level by both Kolmogorov-Smirnov and Mann-Whitney tests. The probability of obtaining a random sample of five bursts with the measured peak fluxes is very low, it varies from 0.02 for SGR 1627-41 to $\sim 10^{-7}$ for SGR 1900+14. In the case of the bursts fluences, the estimated probabilities are in the $0.36-0.05$ range. It should be noted, however, that these values are affected by the high fluences of a number of relatively long "intermediate" bursts (up to tens

\footnotetext{
${ }^{1}$ Two such processes in the framework of the magnetar model have been suggested - small-scale cracking of a NS crust (Thompson \& Duncan 1995) and reconnection in a NS magnetosphere (Lyutikov 2002, 2003)
} 
seconds in duration) which were not excluded from the reference sets.

Supposing there is no intrinsic difference in the bursts emission mechanism, the reasonable explanation of the peculiar brightness of the observed SGR $0501+4516$ bursts can be the relatively lesser distance to the source. Such an interpretation (Kouveliotou et al. 2008) is supported by the localization of the new SGR towards the Galactic anti-center. Without well established natal environments of the known SGRs, their distances are quite uncertain. Various estimations agree in the conservative lower limits of $5-8 \mathrm{kpc}$, with more probable values being closer to $15 \mathrm{kpc}$ for all three known Galactic SGRs (Hurley et al. 1999; Vrba et al. 2000; Corbel et al. 1999; Corbel \& Eikenberry 2004). Soon after the SGR 0501+4516 discovery was found, the proximity of its direction to the young supernova remnant HB9 was reported (Gaensler \& Chatteriee 2008). Assuming a distance of $\sim 1.5 \mathrm{kpc}$ to HB9 (Leahy \& Aschenbach 1995), the peak luminosities of the SGR $0501+4516$ bursts observed by Konus-Wind are estimated to be $(2-5) \times 10^{40} \mathrm{erg} \mathrm{s}^{-1}$. The values of the total energy release, given the same assumptions, amount to $(0.6-6) \times 10^{39} \mathrm{erg}$. In conclusion, these estimations of both parameters are typical for short SGR bursts.

This work was supported by Federal Space Agency of Russia and RFBR grant 09-02-00166a. We gratefully acknowledge an anonymous referee for the detailed comments and suggestions, which have significantly improved this paper. 


\section{REFERENCES}

Aptekar, R. L., et al. 1995, Space Sci. Rev., 71, 265

Aptekar, R. L., et al. 2001, ApJS, 137, 222

Arnaud, K. A. 1996, in ASP Conf. Ser. 101, Astronomical Data Analysis Software and Systems V, ed. G. Jacoby, \& J. Barnes, (San Francisco: ASP), 17

Barthelmy, S. D., et al. 2008, GCN Circular 8113

Corbel, S., et al., 1999, ApJ, 526, L29

Corbel, S., and Eikenberry, S. S., 2004, A\&A, 419, 191

Enoto, T., et al. 2009, ApJ, 693, L122

Fatkhullin, T., et al. 2008, GCN Circular 8160

Fishman, G. J., et al. 2008, GCN Circular 8139

Frederiks, D. D., et al. 2007a, Astronomy Letters, 33, 11, 3

Gaensler, B. M., and Chatterjee, S. 2008, GCN Circular 8149

Göğüss, E., et al. 2000, ApJ, 532, L121

Gogus, E., et al. 2008, GCN Circular 8118

Golentskii, S., Ilinskii, V., \& Mazets, E. 1984, Nature, 307, 41

Golenetskii, S. V., et al. 2008, GCN Circular 8132

Götz, D., et al. 2004, A\&A, 417, L45

Götz, D., et al. 2006, A\&A, 445, 313

Holland, S. T., et al. 2008, GCN Circular 8112

Hurley, K., et al. 1999, ApJ, 510, L111

Kouveliotou, C., et al. 2008, GCN Circular 8138

Krimm, H. A., et al. 2008, GCN Circular 8120

Lyutikov, M. 2002, ApJ, 580, L65

Lyutikov, M. 2003, MNRAS, 346, 540

Leahy, D. A., and Aschenbach, B. 1995, A\&A, 293, 853 
Mazets, E. P., et al. 1979a, Nature, 282, 587

Mazets, E. P., et al. 1979b, Sov. Astron. Lett., 5(6), 343

Mazets, E. P., et al. 1999, ApJ, 519, L151

Mereghetti, S. 2008, A\&ARv, 15, 4, 225

Nakagawa, Y. E., et al. 2007, PASJ, 59, 653

Palmer, D. 2008, Astronomers's Telegram 1678

Palmer, D., et al. 2008, GCN Circular 8150

Rea, N., et al. 2008, GCN Circular 8159

Rea, N., et al. 2008, GCN Circular 8165

Rea, N., et al. 2009, MNRAS accepted (arXiv:0904.2413)

Tanvir, N. R. and Varricatt, W. 2008, GCN Circular 8126

Thompson, C., \& Duncan, R. C. 1995, MNRAS, 275, 255

Vrba, F.J., et al., 2000, ApJ, 533, L17

Woods, P., et al. 1999, ApJ, 527, L47

Woods, P., et al. 2008, GCN Circular 8166 
Table 1. Summary of SGR $0501+4516$ bursts observed by Konus-Wind

\begin{tabular}{|c|c|c|c|c|c|c|}
\hline $\begin{array}{l}\text { Burst }^{\mathrm{a}} \\
\text { date }\end{array}$ & $\begin{array}{l}\mathrm{T}_{0}^{\mathrm{b}} \\
\mathrm{UT}\end{array}$ & $\mathrm{T}_{\text {start }}^{\mathrm{c}}$ & $\begin{array}{l}\text { Duration } \\
\qquad \mathrm{s}\end{array}$ & $\begin{array}{c}\text { Fluence }^{\mathrm{d}} \\
10^{-6} \mathrm{erg} \mathrm{cm}^{-2}\end{array}$ & $\mathrm{~T}_{\text {peak }}{ }^{\mathrm{e}}$ & $\begin{array}{c}\text { Peak flux }{ }^{\mathrm{d}} \\
10^{-4} \mathrm{erg} \mathrm{cm}^{-2} \mathrm{~s}^{-1}\end{array}$ \\
\hline 080823a & $\begin{array}{c}04: 47: 46.194 \\
(04: 47: 48.615)\end{array}$ & -0.064 & 0.752 & $2.32 \pm 0.18$ & -0.002 & $1.08 \pm 0.44$ \\
\hline $080823 \mathrm{~b}$ & $\begin{array}{c}11: 27: 32.652 \\
(11: 27: 35.042)\end{array}$ & -0.068 & 0.446 & $3.72 \pm 0.15$ & -0.004 & $0.62 \pm 0.10$ \\
\hline 080824 & $\begin{array}{c}01: 17: 55.316 \\
(01: 17: 57.640)\end{array}$ & -0.088 & 0.700 & $22.12 \pm 0.68$ & 0.074 & $1.76 \pm 0.17$ \\
\hline 080825 & $\begin{array}{c}04: 48: 27.445 \\
(04: 48: 29.634)\end{array}$ & -0.026 & 0.170 & $2.97 \pm 0.20$ & 0.008 & $1.44 \pm 0.17$ \\
\hline 080826 & $\begin{array}{c}03: 16: 15.017 \\
(03: 16: 17.094)\end{array}$ & -0.166 & 0.514 & $3.74 \pm 0.20$ & 0.030 & $1.12 \pm 0.13$ \\
\hline
\end{tabular}

${ }^{a}$ Bursts 080823a and 080823b were localized by Swift (Palmer et al. 2008a), 080824 and 080825 - by Fermi (Fishman et al. 2008), and 080826 - by Suzaku (Enoto et al. 2009).

${ }^{\mathrm{b}}$ The Konus-Wind trigger time and the corresponding Earth-crossing time (in the brackets)

${ }^{\mathrm{c}}$ The start time of the burst, relative to the trigger time $\mathrm{T}_{0}$

dIn the 20-200 keV range, peak fluxes are on the $2 \mathrm{~ms}$ scale.

eThe start time of the 2-ms peak interval, relative to the trigger time $\mathrm{T}_{0}$ 
Table 2. Summary of spectral fits

\begin{tabular}{lllllll}
\hline \hline \multirow{2}{*}{ Burst } & $\begin{array}{c}\text { Spectra \#\# } \\
\text { (see Fig. 1) }\end{array}$ & $\alpha(\mathrm{CPL})$ & $\begin{array}{c}E_{p}(\mathrm{CPL}) \\
(\mathrm{keV})\end{array}$ & $\begin{array}{c}\chi^{2} / \operatorname{dof}(\mathrm{CPL}) \\
k T_{\text {OTTB }} \\
(\mathrm{keV})\end{array}$ & $\chi^{2} / \operatorname{dof}(\mathrm{OTTB})$ \\
\hline \multirow{2}{*}{$080823 \mathrm{a}$} & 1 & $0.72_{-0.97}^{+0.88}$ & $26.1_{-11.0}^{+6.0}$ & $19.3 / 10$ & $23.6_{-2.5}^{+2.9}$ & $19.6 / 11$ \\
& $1-4$ & $1.42_{-0.76}^{+0.56}$ & $18.6_{-3.5}^{+9.7}$ & $30.6 / 16$ & $24.6_{-2.4}^{+2.6}$ & $31.5 / 17$ \\
\hline $080823 \mathrm{~b}$ & 1 & $1.05_{-1.04}^{+0.92}$ & $22.7_{-8.4}^{+7.5}$ & $11.0 / 10$ & $23.2_{-2.5}^{+2.9}$ & $11.0 / 11$ \\
& $1-4$ & $0.86_{-0.36}^{+0.33}$ & $29.8_{-4.6}^{+3.5}$ & $27.6 / 20$ & $28.1_{-1.3}^{+1.5}$ & $28.1 / 21$ \\
\hline 080824 & 1 & $1.17_{-0.50}^{+0.47}$ & $37.1_{-8.8}^{+6.1}$ & $21.7 / 17$ & $39.1_{-3.3}^{+3.7}$ & $22.1 / 18$ \\
& 2 & $0.71_{-0.40}^{+0.36}$ & $45.3_{-5.1}^{+4.1}$ & $23.8 / 19$ & $41.9_{-2.7}^{+2.9}$ & $25.6 / 20$ \\
& 4 & $1.26_{-0.56}^{+0.50}$ & $27.3_{-7.4}^{+7.4}$ & $24.9 / 15$ & $31.1_{-2.5}^{+2.7}$ & $25.5 / 16$ \\
& $1-4$ & $0.98_{-0.22}^{+0.20}$ & $37.9_{-3.6}^{+3.0}$ & $51.1 / 24$ & $37.7_{-1.5}^{+1.5}$ & $51.2 / 25$ \\
\hline 080825 & 1 & $1.15_{-0.92}^{+0.84}$ & $28.4_{-8.3}^{+7.5}$ & $7.5 / 12$ & $29.9_{-3.4}^{+4.1}$ & $7.6 / 13$ \\
& $1-2$ & $0.48_{-0.65}^{+0.59}$ & $31.6_{-6.4}^{+4.4}$ & $14.3 / 14$ & $26.5_{-2.0}^{+2.1}$ & $16.3 / 15$ \\
\hline 080826 & 1 & $1.00_{-0.54}^{+0.49}$ & $29.5_{-8.9}^{+5.5}$ & $12.1 / 15$ & $29.6_{-2.2}^{+2.3}$ & $12.1 / 16$ \\
& $1-4$ & $0.99_{-0.49}^{+0.45}$ & $28.1_{-7.7}^{+5.1}$ & $13.2 / 19$ & $28.0_{-1.9}^{+2.0}$ & $13.2 / 20$ \\
\hline
\end{tabular}




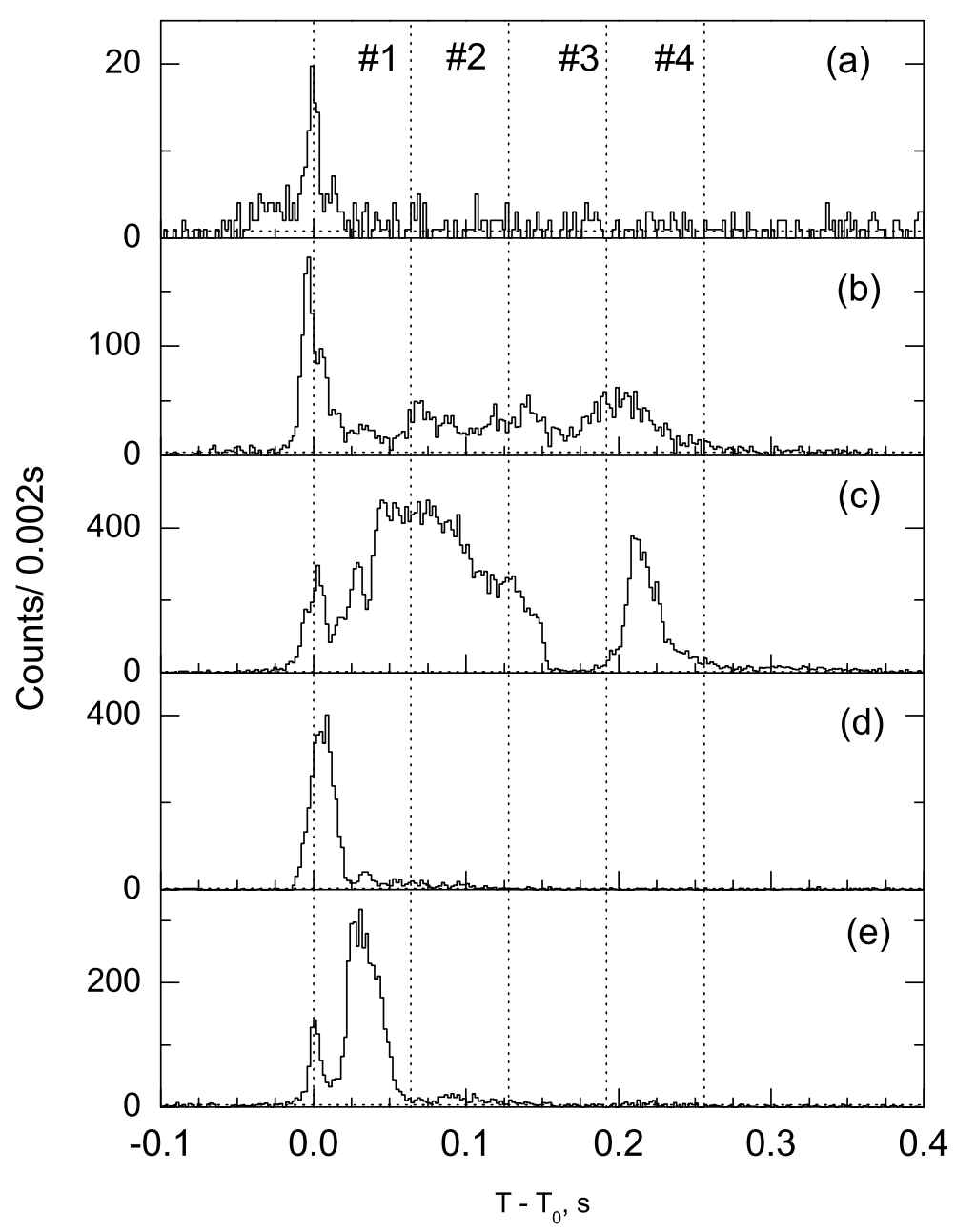

Fig. 1. - The $2 \mathrm{~ms}$ light curves of the five SGR $0501+4516$ bursts detected by Konus-Wind. (a) 080823a (in the 70-300 keV range), (b) 080823b, (c) 080824, (d) 080825, (e) 080826, all in the 20-300 keV range. The trigger times $\mathrm{T}_{0}$ are listed in Table 1 . The vertical dashed lines indicate the four successive 64-ms intervals for which the energy spectra were measured. 


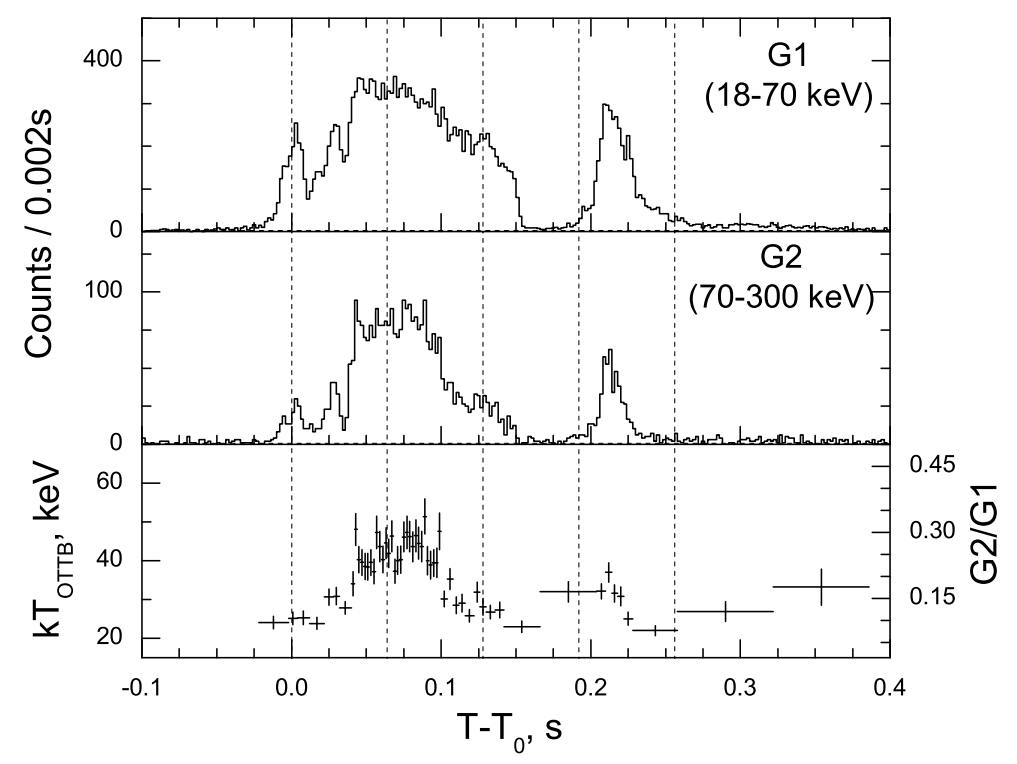

Fig. 2. - The SGR 0501+4516 event detected by Konus-Wind on August 24, 2008. The two upper panels display the count profiles in the available energy windows. The lower panel displays the $k T_{O T T B}$ spectral parameter variation, as derived from the hardness ratio (right axis). A hardnessintensity correlation is clearly apparent. 


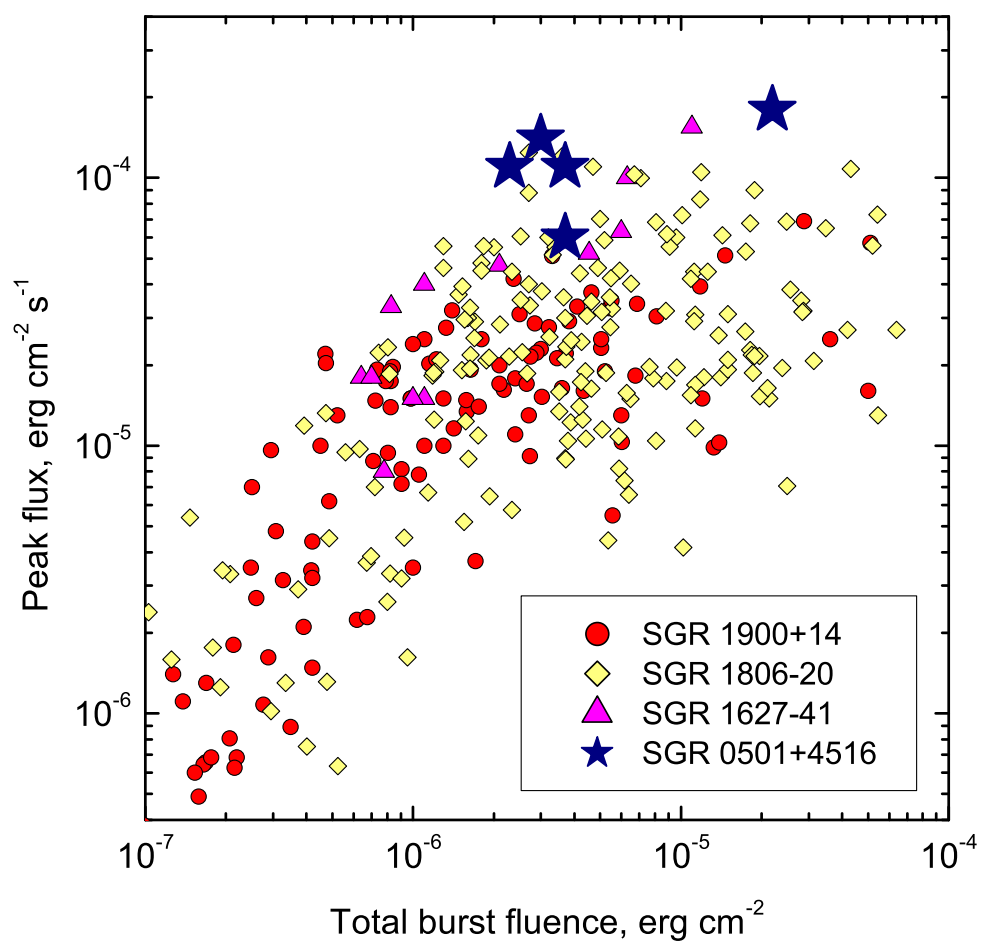

Fig. 3.- The burst energetics, shown with the peak flux vs. the total fluence for each of the events from 4 SGRs: SGR 0501+4516 (marked by stars), selected short bursts detected by Konus-Wind from SGR 1806-20 (diamonds), SGR 1900+14 (circles), and SGR 1627-41 (triangles). All the values are in the 20 to $200 \mathrm{keV}$ range, with the peak fluxes from data on the $2 \mathrm{~ms}$ scale. 